\title{
Gastric wall thickness and stapling in laparoscopic sleeve gastrectomy - a literature review
}

\author{
Krzysztof Barski, Artur Binda, Emilia Kudlicka, Paweł Jaworski, Wiesław Tarnowski \\ Department of General, Oncological and Digestive Tract Surgery, Medical Centre of Postgraduate Education, Orlowski Hospital, \\ Warsaw, Poland
}

Videosurgery Miniinv 2018; 13 (1): 122-127

DOI: https://doi.org/10.5114/wiitm.2018.73362

\begin{abstract}
Despite the growing experience of bariatric surgeons in performing laparoscopic sleeve gastrectomy, the number of complications involving staple line leaks remains constant. Hence a solution to avoid such complications is still sought. A defect of the staple line may be the consequence of an inappropriate choice of staple size in relation to gastric wall thickness. Due to the variable nature of gastric wall thickness, the choice of proper staple height is not obvious. In the few studies in which gastric wall thickness was measured, it was observed to decrease gradually from the antrum to the fundus. However, the authors are divided on the issue of whether gender and body mass index influence gastric wall thickness. The question whether there are other perioperative factors that would allow gastric wall thickness to be predicted remains unanswered.
\end{abstract}

Key words: laparoscopic sleeve gastrectomy, gastric wall thickness, staple height, staple line leak.

\section{Introduction}

The development of laparoscopic techniques and the growing population of morbidly obese patients promote the rapid popularization of bariatric surgery. One of the predominantly performed operations is laparoscopic sleeve gastrectomy (LSG) [1]. It is a restrictive bariatric operation that creates a sleeveshaped stomach along the lesser curvature. This seemingly simple procedure might be mistakenly assumed to be easy to perform for an inexperienced surgeon. Despite the growing number of patients undergoing LSG, surgeons often face complications, with the most serious being staple line leaks [2].

The history of staplers goes back to the $19^{\text {th }}$ century, yet technical errors still occur during surgical procedures involving staplers [3]. One reason for this situation may be the fact that the principal rules of stapler use are not commonly formalized. When in- troducing new devices into the operating room, the surgeon always needs practice to make personal observation and gain experience for the patients' optimal result. The operating surgeon, despite proper theoretical training, is often not able to predict how transected tissue will react to his/her action. It has been pointed out that there is a need for creating a collaborative framework between doctors and engineers to develop science-based protocols describing the proper use of new surgical devices [4].

The steady number of staple line leaks after LSG inspires scientists to find a solution for this complication and parameters to predict it. The dual nature of operated on tissue, which can be divided into liquid and fixed portions, makes it difficult [4]. The balance between these two sections is constantly influenced by the patient's current medical status, age and preoperative treatment. A defect of the staple

Address for correspondence

Artur Binda MD, PhD, Department of General, Oncological and Digestive Tract Surgery, Medical Centre of Postgraduate Education, Orlowski Hospital, 231 Czerniakowska St, 00-416 Warsaw, Poland, phone: +48 6013420 16, +48 225841130 , fax: +48 226227833 , e-mail: quiz0@interia.pl 
line may be the consequence of inappropriate choice of staple size in relation to gastric wall thickness (GWT), inadequate time of tissue precompression, or inappropriate pressure of the firing stapler. The search for the answer is still in progress. Only a few studies have analyzed GWT from the perspective of bariatric surgery.

The objective of this review was to analyze the available literature on the issue of GWT and its influence on staple line integrity.

\section{Stapling devices and techniques}

Most bariatric procedures are currently performed with mechanical stapling devices. Matching the staple height with the thickness of transected and sutured tissue is crucial to perform successful surgery. Staples deployed from the cartridge should compress the tissue to provide proper hemostasis and impermeable anastomosis without causing ischemia and tissue necrosis. Over the years a series of staplers have been invented and improved to reduce the risk of staple line integrity.

There are two main manufacturers providing staplers used for LSG: Covidien-Medtronic's Endo GIA with Tri-Staple Technology, and Ethicon's Echelon Flex GST System. Each of these staplers is reloaded with cartridges containing staples that differ in height and are thereby dedicated to various tissue thicknesses $[5,6]$. The size of the staples in cartridges is indicated by color (Tables I and II).

Manufacturers request the operator to match the used cartridge with the anticipated thickness of the transected tissue $[5,6]$. The choice of the reload used by the operating surgeon should correspond to the thickness and histological features of the transected organ wall so that the desired, closed, B-shaped staple is formed. Improper choice may lead to malformation of the closed staples and may have life-threatening consequences of staple line leak.

Covidien's Endo GIA stapler with Tri-Staple Technology is reloaded with 4 types of cartridges of varying height and three rows of staples. The height of the staples decreases inwards. Each of the reloads is intended to be used over a different tissue thickness range. The gray reload is for tissue ranging from 0.75 to $0.88 \mathrm{~mm}$. The tan reload is intended to be used on vascular/medium tissue of 0.88 to $1.88 \mathrm{~mm}$ thickness. The purple reload is for medium/thick tissue ranging from 1.5 to $2.25 \mathrm{~mm}$. The black reload should be used on very thick tissue ranging from 2.25 to $3.0 \mathrm{~mm}$. The reloads are available in three lengths: 30, 45 and $60 \mathrm{~mm}$ [5].

Echelon Flex works with 5 kinds of reloads, also differing in intended tissue thickness. The white reload should be used on the thinnest tissue. The blue reload is for tissue of 1.25 to $1.75 \mathrm{~mm}$ thickness. Gold works on tissue thickness between 1.5 to $3.0 \mathrm{~mm}$. Green is intended to be used on tissue between 1.75 to $3.25 \mathrm{~mm}$ thick. Black should be used on the thickest tissue [6].

\section{Biomechanical obstacles}

Despite the fact that the cause of formed staple line imperfection is a source of major bariatric complications, there are not many papers analyzing the biology of this issue.

Table I. Open staple height and range of tissue thickness for cartridges produced by Covidien

\begin{tabular}{|lcccc|}
\hline \multicolumn{5}{c}{ Endo GIA Tri-Staple Technology (Covidien) } \\
\hline Cartridge & Black & Purple & Tan & Gray \\
\hline Open staple height $[\mathrm{mm}]$ & $4-4.5-5$ & $3-3.5-4$ & $2-2.5-3$ & $2-2-2$ \\
\hline Range of tissue thickness $[\mathrm{mm}]$ & $2.25-3.0$ & $1.5-2.25$ & $0.88-1.8$ & $0.75-0.88$ \\
\hline
\end{tabular}

Table II. Open staple height and range of tissue thickness for cartridges produced by Ethicon

\begin{tabular}{|lccccc|}
\hline \multicolumn{5}{|c|}{ Echelon Flex (Ethicon) } \\
\hline Cartridge & Black & Green & Gold & Blue & White \\
\hline Open staple height $[\mathrm{mm}]$ & 4.2 & 4.1 & 3.8 & 3.6 & 2.6 \\
\hline Range of tissue thickness $[\mathrm{mm}]$ & $2.3-4.0$ & $2.0-3.25$ & $1.8-3.0$ & $1.5-1.75$ & $1.0-2.0$ \\
\hline
\end{tabular}


The study of tissue biomechanics by Fung was summarized from the perspective of stapling by Baker $[7,8]$. He claims that under stapler compression three main processes occur: tissue creep, stress relaxation and tensile stress. The essence of the matter is to maintain a balance between these three reactions, and the main modifiable variable is duration of stapling pressure on operated on tissue.

The duration of the pressure applied by the stapler on the tissue before firing is called precompression. In his report from 1967 Astafiev claims that after 15-20 s of pressure applied by the stapler, the tissue thickness reaches its maximal compression before its histological structure is damaged. This observation has become the standard for all reports on tissue thickness measurements [9]. Nakayama examined how precompression affects the formation of the staple line. In his research, optimal B-shaped staples were more frequently formed when the precompression was longer ( 5 min vs. 1 min, $p<0.001$; $1 \mathrm{~min}$ vs. 0 min, $p=0.001$ ). This research suggests that one should wait some time between placing the tissue in the stapler and firing [10].

Chekan highlights the variable nature of the operated on organ wall thickness, which can be influenced by location, sex, age and preoperative features. Adequate compression of the tissue is dependent on these factors. Proper compression allows one to create optimal formation of closed staples, preventing the development of ischemia while still ensuring hemostasis [4].

All of the tissue thickness studies refer to the paper by Astafiev from 1967 [9]. The researcher conducted a series of simulations to obtain the optimal compression of the stapling devices during anastomosis formation. In this analysis, compression of 8 grams per square millimeter $\left(\mathrm{g} / \mathrm{mm}^{2}\right)$ during creation of anastomosis was determined as best to maintain hemostasis and avoid ischemia [11]. The author also claimed that during stapling of the intestines and the esophagus less compression is required $\left(6 \mathrm{~g} / \mathrm{mm}^{2}\right)$.

Baker conducted a series of simulations to understand tissue physiology under stapler compression [8]. In his paper two main groups of causes were distinguished, mechanical and ischemic, both caused by the intraluminal pressure exceeding the closed staples strength. More often the leak has a mechanical etiology, occurring usually in the first 2 postoperative days, in contrast to an ischemic cause, which is much less frequent and occurs 5-7 days postoperatively.

\section{Gastric wall thickness studies}

Many studies on the occurrence of anastomosis leaks underline the importance of surgeons' knowledge of the thickness of the operated on organ's wall. However, only four publications focus on this issue [11-14].

The first author to measure the thickness of the operated on gastric wall from the point of view of stapling and bariatric procedures was Hazem Elariny. In his study he enrolled 50 patients (37 females, 13 males) with a mean body mass index (BMI) of $50 \mathrm{~kg} /$ $\mathrm{m}^{2}$ and a mean age of 41.8 years. The thickness of the excised gastric specimen was measured at six positions along the staple line. Statistical analysis indicated that gender and location have an influence on tissue thickness, with the thickest tissue in the pyloric region and the thinnest in the fundus. The authors estimated the thickness of the stomach for males at the $97.5^{\text {th }}$ percentile to be $4.9 \mathrm{~mm}$ [11].

Rawlins measured 50 resected gastric sleeves of patients with mean BMl $49 \mathrm{~kg} / \mathrm{m}^{2}$ and average age 42 years ( 40 females and 10 males). The results confirmed that thickness is significantly different at each location. Male gender is associated with a thicker gastric wall but only in the antrum. The authors observed that BMI has an influence on gastric wall thickness but only in patients with $\mathrm{BMI}$ over $50 \mathrm{~kg} / \mathrm{m}^{2}[12]$.

Van Rutte measured the thickness of resected gastric sleeves at 5 points along the staple line, in 33 patients (26 females, 7 males), with mean age 42 years and mean BMI $45 \mathrm{~kg} / \mathrm{m}^{2}$. The authors took the measurements after flattening the gastric folds and thereby obtained full contact of the gastric layers during compression. Based on this observation, the authors claim that excessive pressure is applied by the staplers, which may be the cause of gastric wall tearing [13].

In their study Rose Huang and Michel Gagner proposed that for better stapler reload selection a new intraoperative technology should be invented to obtain the GWT measurement before applying the stapler. They analyzed the GWT of 26 patients (15 females, 11 males), with mean BMI $45.3 \mathrm{~kg} / \mathrm{m}^{2}$. Based on their findings, they suggest that a stapler that exceeds the range of the black cartridge should 
Table III. Gastric wall thickness measurements taken at three anatomical regions of the stomach summarized from four publications

\begin{tabular}{|c|c|c|c|c|c|}
\hline \multicolumn{2}{|c|}{ Parameter } & \multicolumn{4}{|c|}{ Author (year of publication) } \\
\hline & & Elariny (2005) & Rawlins (2014) & Van Rutte (2015) & Huang (2015) \\
\hline \multirow[t]{3}{*}{ Female } & Fundus $[\mathrm{mm}]$ & 1.61 & 1.94 & 1.37 & 1.72 \\
\hline & Corpus $[\mathrm{mm}]$ & 2.34 & 2.32 & 1.98 & 2.64 \\
\hline & Antrum $[\mathrm{mm}]$ & 3.09 & 2.64 & 2.55 & 3.09 \\
\hline \multirow[t]{3}{*}{ Male } & Fundus $[\mathrm{mm}]$ & 1.81 & 2.09 & 1.37 & 1.67 \\
\hline & Corpus [mm] & 2.6 & 2.38 & 1.98 & 2.57 \\
\hline & Antrum $[\mathrm{mm}]$ & 3.17 & 2.96 & 2.55 & 3.12 \\
\hline
\end{tabular}

Table IV. Influence of location, gender and BMI on gastric wall thickness

\begin{tabular}{|c|c|c|c|c|c|}
\hline \multirow[t]{2}{*}{ Parameter } & & \multicolumn{4}{|c|}{ Author } \\
\hline & & Elariny (2005) & Rawlins (2014) & Van Rutte (2015) & Huang (2015) \\
\hline \multirow{3}{*}{$\begin{array}{l}\text { Influence } \\
\text { on gastric } \\
\text { wall } \\
\text { thickness }\end{array}$} & Location & Yes & Yes & Yes & Yes \\
\hline & Gender & Yes & Yes & Not analyzed & $\begin{array}{c}\text { Depends } \\
\text { on localization }\end{array}$ \\
\hline & BMI & No & Yes (over $50 \mathrm{~kg} / \mathrm{m}^{2}$ ) & No & Not analyzed \\
\hline
\end{tabular}

be introduced, and concluded that at present there is no universal method to predict GWT 14].

In all the presented studies GWT was found to differ according to the location. Similar results were obtained within three regions of the stomach. The thinnest wall is located in the fundus, the thickest in the antrum (Table III).

The researchers tried to determine how demographic features of the patients influence GWT. Gender is a factor affecting GWT according to three of four papers. Male patients tend to have a thicker gastric wall at all locations according to two of four papers. In one study BMI was found to influence GWT only when it was over $50 \mathrm{~kg} / \mathrm{m}^{2}$ [12]. In other papers BMI had no significance in relation to GWT (Table IV).

The above-mentioned authors investigated the same issue, but there is a dissimilarity in the measuring devices used in their studies. Van Rutte et al. draws attention to the need for flattening the mucosa folds before measuring the tissue thickness, and questions the choice of the optimal measuring method. In his research, a special electronic thickness gauge was developed to allow adjustment of optimal pressure during each measurement [13].
Other studies used thickness measurement devices based on information from the publication by Astafiev about optimal pressure on the tissue during the use of staplers [9].

\section{Discussion}

With the increasing popularity of metabolic procedures, weight loss surgery is becoming a separate field of specialization for surgeons. The quickly growing number of stapler types and staple line reinforcement techniques creates a "knowledge gap" about how the potential of these devices may be utilized during bariatric procedures.

Gastric sleeve leaks are still the most dangerous possible postoperative surgical complication after LSG. According to Stroh et al., they may occur in up to $1.5 \%$ of patients operated on [2]. Many authors underline the lack of a clear recommendation on the staple height that should be applied during LSG. In a study by Major et al. it was found that a greater number of stapler firings increases the risk of postoperative complications after LSG, which underlines the necessity of introducing intraoperative guidelines on the use of staplers [15]. In the International Sleeve Gastrectomy Expert Panel Consensus from 2012 it 
was stated that recommendations on staple height were only partially established. It was decided that when performing LSG to the level of the incisura angularis at least green cartridges should be used, while nothing less than blue should be used from the incisura angularis to the angle of His. Revision should be performed with at least green cartridges [16].

Precise information about GWT would allow better staple line integrity to be achieved and therefore reduce the rate of gastric sleeve leaks. Gastric wall thickness is a feature that varies among patients but is still measurable. There are some publications about GWT measured preoperatively by means of ultrasonography (USG) and computed tomography (CT). Yazar et al., in the evaluation of GWT measured in USG in patients qualified for sleeve gastrectomy, concluded that GWT is not associated with gender or BMI but is increased in patients with gastritis [17]. It must be emphasized that there is a major difference between GWT observed by means of USG in this study (range: 5.3-15.8) and the measurements of excised gastric specimens in the studies presented in Table IV. In a study conducted by Pickhardt and Asher, a CT scan of 153 patients revealed that the thickness of the gastric antral wall had a range between 1.8 and $12 \mathrm{~mm}$ overall (when measuring the anterior and posterior wall separately), which is also very different from the results measured on resected specimens [18]. In a study designed by Larsen, GWT measured by means of endoscopic ultrasound had no correlation with $\mathrm{BMI}$, and no difference between the antrum, body and fundus was observed. In this analysis the GWT range was 3-4 $\mathrm{mm}$, which is similar to measurements of excised gastric specimens [19]. These findings suggest that only preoperative endoscopic ultrasound may be helpful for the surgeon in adjusting the staple height to the thickness of the transected tissue.

In 10 years only 4 studies have focused on the measurement of GWT based on the excised gastric specimen. The results of these studies allow the surgeon to predict that GWT decreases gradually from the antrum to the fundus, and that it is thicker in male patients [11-14]. However, the authors are divided on the issue of whether gender and BMI have an influence on GWT. It remains unanswered whether there are other perioperative factors that would allow one to predict GWT more precisely and consequently reload the stapler with the proper cartridge. It has been proven in another bariatric procedure - laparoscopic gastric bypass - that adequate choice of staple height may improve the postoperative outcome [20]. The decision made on cartridge selection should be based on a universal protocol, which may be helpful in preventing potential lawsuits. Constant development of new methods for preventing gastric leaks, and controversies about oversewing of the staple line prove that studies of gastric wall biology from the perspective of bariatric surgery should be thoroughly analyzed [21]. It is especially important given that there are no clear guidelines on how to manage the leaks after sleeve gastrectomy, and the treatment outcomes are not always good [22].

\section{Conclusions}

Matching the staple height with the thickness of transected tissue is essential to perform successful weight reduction surgery. A sufficient amount of precompression time is key to improving patient outcomes and proper staple formation. Accurate determination of GWT before stapler firing would allow life-threatening complications to be avoided. Precise recommendations on the selection of the appropriate stapler cartridge have not been established. To achieve a universal consensus further analysis on the biology of GWT should be conducted.

\section{Conflict of interest}

The authors declare no conflict of interest.

\section{References}

1. Chang SH, Stoll CR, Song J, et al. Bariatric surgery: an updated systematic review and meta- analysis, 2003-2012. JAMA Surg 2014; 149: 275-87.

2. Stroh C, Kockerling F, Volker L, et al. Obesity Surgery Working Group, Competence Network Obesity. Results of more than 11,800 sleeve gastrectomies: data analysis of the German Bariatric Surgery Registry. Ann Surg 2016; 263: 949-55.

3. Offodile AC, Feingold DL, Nasar A, et al. High incidence of technical errors involving the EEA circular stapler: a single institution experience. J Am Coll Surg 2010; 210: 331-5.

4. Chekan E, Whelan RL, Feng AH. Device-tissue interactions: a collaborative communications system. Ann Surg Innov Res 2013; 7: 10.

5. Endo GIATM Reloads with Tri-Staple ${ }^{T M}$. Technology Technical Brochure. Available at: www.medtronic.com/content/dam/covidien/ library/us/en/product/surgical-stapling/endo-gia-reloadstechnical-brochure.pdf; Accessed: 9 Feb 2017.

6. GST Bariatric Brochure. Available at: www.ethicon.com/sites/ default/files/managed-documents/030235-150225_gst-bariatric-brochure.pdf; Accessed: 9 Feb 2017. 
7. Fung YC. Biomechanics: Mechanical Properties of Living Tissues. $2^{\text {nd }}$ ed. Springer Verlag, New York 1993.

8. Baker RS, Foote J, Kemmeter P, et al. The science of stapling and leaks. Obes Surg 2004; 14: 1290-8.

9. Astafiev G. Investigation process relating to tissue compression in suturing and stapling apparatus. Surgical Staplers (Chirurgicheskiye Shivayushiye Apparaty) 1967; 7: 22-31.

10. Nakayama S, Hasegawa S, Nagayama S, et al. The importance of precompression time for secure stapling with a linear stapler. Surg Endosc 2011; 25: 2382-6.

11. Elariny $\mathrm{H}$, Gonzalez H, Wang B. Tissue thickness of human stomach measured on excised gastric specimens from obese patients. Surg Technol Int 2005; 14: 119-24.

12. Rawlins L, Rawlins M, Teel D. Human tissue thickness measurements from excised sleeve gastrectomy specimens. Surg Endosc 2014; 28: 811-4.

13. Van Rutte PW, Naagen BJ, Spek M, et al. Gastric wall thickness in sleeve gastrectomy patients: thickness variation of the gastric wall. Surg Technol Int 2015; 27: 123-8.

14. Huang R, Gagner M. A thickness calibration device is needed to determine staple height and avoid leaks in laparoscopic sleeve gastrectomy. Obes Surg 2015; 25: 2360-7.

15. Major P, Wysocki M, Pędziwiatr M, et al. More stapler firings increase the risk of perioperative morbidity after laparoscopic sleeve gastrectomy. Videosurgery Miniinv 2017; https://doi. org/10.5114/wiitm.2017.70197.

16. Rosenthal R. International Sleeve Gastrectomy Expert Panel. International Sleeve Gastrectomy Expert Panel Consensus Statement: best practice guidelines based on experience of $>12,000$ cases. Surg Obes Relat Dis 2012; 8: 8-19.

17. Yazar FM, Baykara M, Karaağaç M, Bülbüloğlu E. The role of conventional ultrasonography in the evaluation of antrum wall thickness in obese patients. Obes Surg 2016; 26: 2995-3000.

18. Pickhardt PJ, Asher DB. Wall thickening of the gastric antrum as a normal finding: multidetector CT with cadaveric comparison. AJR Am J Roentgenol 2003; 181: 973-9.

19. Larsen MC, Yan BM, Morton J, Van Dam J. Determination of the relationship between gastric wall thickness and body mass index with endoscopic ultrasound. Obes Surg 2011; 21: 300-4.

20. Nguyen NT, Dakin G, Needleman B, et al. Effect of staple height on gastrojejunostomy during laparoscopic gastric bypass: a multicenter prospective randomized trial. Surg Obes Relat Dis 2010; 6: 477-82.

21. Kwiatkowski A, Janik MR, Paśnik K, Stanowski E. The effect of oversewing the staple line in laparoscopic sleeve gastrectomy: randomized control trial. Videosurgery Miniinv 2016; 11: 149-55.

22. Špička P. Staple line leak with peritonitis after laparoscopic sleeve gastrectomy - a solution in one to six steps. Videosurgery Miniinv 2017; 12: 154-9.

Received: 19.11.2017, accepted: 14.01.2018. 